\title{
UMA ANÁLISE DAS POTENCIALIDADES DOS APLICATIVOS MATHUP, AVENTURA DO BEBÊ PANDA COM MATEMÁTICA E TRAIN BRAIN PARA O ENSINO DAS OPERAÇÕES BÁSICAS NA EDUCAÇÃO INFANTIL E ANOS INICIAIS DO ENSINO FUNDAMENTAL
}

\author{
AN ANALYSIS OF THE POTENTIALITIES OF MATHUP APPLICATIONS, \\ ADVENTURE OF THE BABY PANDA WITH MATHEMATICS AND TRAIN BRAIN \\ FOR THE TEACHING OF BASIC OPERATIONS IN CHILDHOOD EDUCATION \\ AND INITIAL YEARS OF FUNDAMENTAL EDUCATION
}

\author{
Ewellyn Amâncio Araújo Barbosa ${ }^{1}$; Sidney Leandro da Silva Viana ${ }^{2}$, Claudia de \\ Oliveira Lozada ${ }^{3}$
}

\begin{abstract}
RESUMO
A utilização dos dispositivos móveis como os smartphones e tablets é cada vez mais crescente na sociedade. A partir disso, o uso de aplicativos está cada vez mais evidente no dia-a-dia dos indivíduos, se fazendo necessário adaptar o próprio processo educativo para a inserção desses recursos tecnológicos, promovendo uma maior aproximação entre os conteúdos ministrados em sala de aula e o próprio aluno. Nessa perspectiva, esse trabalho tem como objetivo discutir as potencialidades de novas tecnologias para o ensino de Matemática, a partir de uma análise dos aplicativos MathUp, Aventura do Bebê Panda com Matemática e o Train Brain enquanto ferramentas que auxiliam no desenvolvimento das habilidades matemáticas que estão previstas na Base Nacional Comum Curricular, como o raciocínio lógico, cálculo mental e agilidade. Para tanto, por meio de uma pesquisa qualitativa, relacionamos as potencialidades desses aplicativos com o referencial teórico que apresenta a importância do uso de novas tecnologias em sala de aula e o impacto que essas tecnologias apresentam no processo de ensino-aprendizagem. A partir dessa análise, podemos concluir que os aplicativos são potencialmente significativos para estimular o desenvolvimento das habilidades matemáticas citadas e constituem ferramentas auxiliares para o ensino de operações matemáticas, nos quais os alunos poderão aprender de forma dinâmica a manuseá-los para realizar as operações e desenvolver estratégias de resolução, enfatizando os

\footnotetext{
${ }^{1}$ Licenciada em Matemática. Mestranda em Ensino de Ciências e Matemática. Universidade Federal de Alagoas, Maceió, Alagoas, Brasil. Endereço para correspondência: Av. Lourival Melo Mota, S/N, Tabuleiro do Martins, Maceió, Alagoas, Brasil. E-mail: ewellynbsantos@gmail.com

(iD ORCID iD: https://orcid.org/0000-0001-6536-6159.

${ }^{2}$ Licenciado em Matemática. Mestrando em Ensino de Ciências e Matemática. Universidade Federal de Alagoas, Maceió, Alagoas, Brasil. Endereço para correspondência: Av. Lourival Melo Mota, S/N, Tabuleiro do Martins, Maceió, Alagoas, Brasil. E-mail: sidneylviana@icloud.com

iD ORCID iD: https://orcid.org/0000-0002-5309-486X

${ }^{3}$ Doutora em Educação (Linha de Pesquisa em Ensino de Ciências e Matemática) - Universidade de São Paulo (USP). Docente da Universidade Federal de Alagoas, Maceió, Alagoas, Brasil. Endereço para correspondência: Av. Lourival Melo Mota, S/N, Tabuleiro do Martins, Maceió, Alagoas, Brasil. E-mail: clalloz@yahoo.com.br
}

iD ORCID iD: https://orcid.org/0000-0003-1425-9956 
conteúdos procedimentais como colocado por Zabala (1998). Deste modo, os alunos poderão partir de um ensino focado na oralidade e escrita para um ambiente no qual eles possam interagir de modo mais dinâmico com os conteúdos matemáticos utilizando os aplicativos, desenvolvendo o pensamento aritmético, além de estratégias ágeis e que serão facilmente lembradas posteriormente, bem como de poderem acessar os aplicativos em outros momentos e espaços, além da escola.

Palavras-chave: Ensino de Matemática; Aplicativos; Potencialidades.

\begin{abstract}
The use of mobile devices such as smartphones and tablets is increasing in society. From this, the use of applications is increasingly evident in the daily lives of individuals, making it necessary to adapt the educational process itself for the insertion of these technological resources, promoting a closer relationship between the contents taught in the classroom and the student himself. In this perspective, this work aims to discuss the potential of new technologies for the teaching of Mathematics, from an analysis of the applications MathUp, Baby Panda Adventure with Mathematics and Train Brain as tools that help in the development of mathematical skills that are foreseen in the National Common Curricular Base, such as logical reasoning, mental calculation and agility. Therefore, through a qualitative research, we relate the potential of these applications to the theoretical framework that presents the importance of using new technologies in the classroom and the impact that these technologies have on the teaching-learning process. From this analysis, we can conclude that the applications are potentially significant to stimulate the development of the mathematical skills mentioned and constitute auxiliary tools for teaching mathematical operations, in which students can dynamically learn how to handle them to perform operations and develop resolution strategies, emphasizing the procedural contents as stated by Zabala (1998). In this way, students will be able to move from teaching focused on orality and writing to an environment in which they can interact more dynamically with mathematical content using applications, developing arithmetic thinking, as well as agile strategies that will be easily remembered later, as well as being able to access the applications at other times and spaces, in addition to the school.
\end{abstract}

Keywords: Mathematics teaching; Apps; Potentialities. 


\section{Introdução}

Ao longo dos anos, nota-se que as discussões sobre as práticas docentes vem causando um impacto positivo no que se refere às reflexões sobre o ensino e de como o professor pode auxiliar no processo de construção do conhecimento, porém é necessário que essas discussões não fiquem presas em um documento eletrônico ou impresso, mas que essas reflexões possam mudar de fato o modo com que o professor ministra suas aulas, podendo inovar em suas metodologias de ensino e, desta forma, possam contribuir para um melhor aprendizado (ALBINO, 2015).

Na sociedade atual, sabe-se que as tecnologias digitais estão inseridas em diversos campos, seja no trabalho, na rua, em casa, no trânsito, inclusive na área educacional. A Base Nacional Comum Curricular (BRASIL, 2018) considera importante o uso das tecnologias digitais no contexto escolar, designando ações que devem ser tomadas para que os alunos possam ser inseridos na cultura digital. Para isso, o professor deverá incluir em suas aulas, ferramentas pedagógicas através das tecnologias digitais com o intuito de promover uma aprendizagem mais dinâmica e significativa de determinado conteúdo (EYYAM; YARATAN, 2014).

Muitos estudantes apresentam diversas dificuldades na compreensão de conteúdos matemáticos e isto decorre por diversos fatores, dentre eles, o método de ensino adotado pelo professor e a utilização de poucos recursos que auxiliem na construção do conhecimento.

Diante disto, o uso de tecnologias em sala de aula é de grande valia e se faz necessário (D’AMBRÓSIO, 1996), bem como o uso de aplicativos com foco em conteúdos que podem apresentar uma abordagem mais atrativa para ajudar na compreensão dos conceitos quando utilizados em sala de aula, fazendo com que a inserção da tecnologia no contexto escolar provoque mudanças significativas no processo ensino-aprendizagem, conforme coloca Moran (2013).

Sendo assim, este trabalho apresenta uma análise das potencialidades de três aplicativos - MathUp, Aventura do Bebê Panda com Matemática e Train Brain - que podem ser utilizados em sala de aula como ferramenta pedagógica de modo a contribuir para a aprendizagem e assimilação de conteúdos matemáticos. 


\section{As tecnologias no ensino de Matemática}

Segundo o principal documento norteador da Educação Básica no Brasil, a Base Nacional Comum Curricular, a BNCC (BRASIL, 2018), as tecnologias digitais constituem um fator importante no estímulo ao desenvolvimento de competências e habilidades dos alunos.

Para tal, a BNCC (BRASIL, 2018) orienta que os alunos sejam inseridos na cultura digital seguinto três pilares: alfabetização digital, em que o aluno deve conhecer, compreender e fazer uso básico dos recursos tecnológicos; o letramento digital, em que os estudantes devem conseguir analisar, avaliar, aplicar e criar conteúdos ou recursos utilizando essas tecnologias e a fluência digital, que é quando a tecnologia é incorporada de modo natural no processo de ensino e aprendizagem (OSMUNDO, 2018).

É nesse sentido que surge mais um desafio para o docente que, agora, deve incorporar à sua prática profissional os recursos tecnológicos visando proporcionar um ambiente de aprendizagem em que o aluno adquira domínio no uso desses recursos no seu cotidiano e também utilize esses recursos como ferramenta de aprendizagem na sala de aula e também fora dela. Essa importância de se incorporar a utilização de recursos tecnológicos nas práticas docentes ficou bastante evidente em 2020 durante a pandemia em que as aulas presenciais foram suspensas e adotou-se o ensino remoto.

O uso das tecnologias para o ensino de Matemática vai além do simples fato de tornar o ensino de aprendizagem como um processo que transcende a escrita e oralidade, visando construir uma interação entre os alunos e os instrumentos digitais para a construção do conhecimento, como também a necessidade de substituir os processos que priorizam somente o ensino pautado na exposição, num receber passivo dos conteúdos por parte dos alunos, sem que eles possam participar efetivamente da construção dos conceitos (D’AMBRÓSIO, 1996) para um processo ensino-aprendizagem mais dinâmico e com participação ativa dos alunos.

Nesse sentido, não se pode negar a influência que os dispositivos tecnológicos exercem atualmente na sociedade e, em particular, o smartphone que apresenta muitos recursos e funcionalidades a serem utilizados no ambiente educativo, como calculadora, conversor de moeda, dicionários, plataformas educativas como o Google Sala de Aula, até a possibilidade de fazer download, de outras tecnologias, os chamados aplicativos. 
Nessa perspectiva, resgatamos um preceito fundamental colocado pelos Parâmetros Curriculares Nacionais (BRASIL, 1998), os PCNs, que sugeria a utilização de recursos variados para o ensino, dentre eles, os tecnológicos como os computadores:

\begin{abstract}
A utilização de materiais diversificados como jornais, revistas, folhetos, propagandas, computadores, calculadoras, filmes, faz o aluno sentir-se inserido no mundo à sua volta.[...] É indiscutível a necessidade crescente do uso de computadores pelos alunos como instrumento de aprendizagem escolar, para que possam estar atualizados em relação às novas tecnologias da informação e se instrumentalizarem para as demandas sociais presentes e futuras. (BRASIL, 1998, p. 67).
\end{abstract}

Essa mesma linha é seguida pela Base Nacional Comum Curricular (BRASIL, 2018) que promove a utilização das TDICs no processo ensino-aprendizagem, colocandoas na competência geral:

Compreender, utilizar e criar tecnologias digitais de informação e comunicação de forma crítica, significativa, reflexiva e ética nas diversas práticas sociais (incluindo as escolares) para se comunicar, acessar e disseminar informações, produzir conhecimentos, resolver problemas e exercer protagonismo e autoria na vida pessoal e coletiva.

A partir dessas colocações dos documentos curriculares, podemos entender que quando falamos de novas tecnologias aplicadas à Educação não devemos somente nos remeter ao computador, devendo pensar de forma mais ampla, incluindo as tecnologias móveis, assim como os softwares e aplicativos que também desempenham um papel importante no processo ensino-aprendizagem:

\footnotetext{
O conceito de tecnologia educacional pode ser enunciado como o conjunto de procedimentos (técnicas) que visam 'facilitar' os processos de ensino e aprendizagem com a utilização de meios (instrumentais, simbólicos ou organizadores) e suas consequentes transformações culturais. (REIS, 2010, p.4)
}

Ainda sobre a importância dessas tecnologias para o ensino de Matemática, destacamos uma fala de D’Ambrósio (1996, p. 13):

Ao longo da evolução da humanidade, Matemática e tecnologia se desenvolveram em íntima associação, numa relação que poderíamos dizer simbiótica. A tecnologia entendida como convergência do saber (ciência) e do fazer (técnica), e a matemática são intrínsecas à busca solidária do sobreviver 
e de transcender. A geração do conhecimento matemático não pode, portanto ser dissociada da tecnologia disponível.

Nesse sentido, destacam-se os aplicativos que são softwares que realizam tarefas específicas, e que estão presentes nas tecnologias móveis como os smartphones, e que se utilizados da forma correta podem apresentar-se como um instrumento potencialmente significativo para o ensino de Matemática.

Os aplicativos de um modo geral estimulam o interesse dos alunos pelos conteúdos matemáticos (ALMEIDA, 2016), apresentam uma interface interativa e muitas vezes lúdica, ajudam a desenvolver o raciocínio lógico, a percepção de regularidades matemáticas, além de auxiliar o aluno na busca de estratégias para a resolução de problemas, permitindo que eles possam levantar, investigar e avaliar hipóteses, promovendo o desenvolvimento dos processos cognitivos (KYRIAKIDES, MELETIOUMAVROTHERIS e PRODROMOU, 2016).

Lorenzoni (2016) pontua que a tecnologia móvel permite que qualquer pessoa utilize o conteúdo que é disponibilizado, tornando o processo de ensino mais fácil de ser manuseado pelo próprio aluno, e além do mais amplia o tempo e os espaços onde pode ser utilizada, além da escola e dos horários regulares de aula.

Moretto (2015, p. 34) coloca que as tecnologias móveis englobam "quaisquer equipamentos ou periféricos tecnológicos que podem ser facilmente transportados (...) que cabem na palma das mãos das pessoas, que acessam e levam informação para qualquer lugar." A autora pontua que as tecnologias móveis integram o grupo das mídias interativas, pois promovem interatividade, mobilidade e portabilidade e desencadeiam a aprendizagem móvel, também chamada de m-learning.

Moretto (2015) ainda explica que a aprendizagem móvel envolve três paradigmas: um modelo flexível de aprendizagem (pois os alunos podem acessar os conteúdos em tempos e espaços diversos aos da escola), padrão pedagógico apoiado em dispositivos tecnológicos sem fios (necessitando apenas de wi-fi) e diretrizes voltadas para a aprendizagem centrada no aluno (o aluno é protagonista de sua própria aprendizagem).

Como resultado dessa difusão dos aplicativos educacionais a partir de experiências educativas inovadoras, tem-se o fato de que tanto o aluno quanto o professor, a escola e a própria sociedade se beneficiam, uma vez que os aplicativos são capazes de 
transformar o saber e as práticas pedagógicas de ensino consideradas tradicionais, auxiliando o aluno na assimilação de conteúdos através uma dinâmica mais interativa, conforme coloca Moran (2013, p. 30):

\begin{abstract}
As tecnologias digitais móveis provocam mudanças profundas na educação presencial e a distância. Na presencial, desenraizam o conceito de ensinoaprendizagem localizado e temporalizado. Podemos aprender desde vários lugares, ao mesmo tempo, on e off-line, juntos e separados. Na educação a distância permitem o equilíbrio entre a aprendizagem individual e a colaborativa, de forma que os alunos de qualquer lugar podem aprender em grupo, em rede, da forma flexível e adequada para cada aluno (MORAN, 2013, p. 30).
\end{abstract}

A partir desse breve referencial teórico, por meio de uma pesquisa qualitativa (BOGDAN e BIKLEN, 1994), analisamos as potencialidades de três aplicativos para o ensino de Matemática

A seleção dos aplicativos considerou os seguintes aspectos: abrangência (Educação Infantil e Ensino Fundamental considerando que são níveis de ensino nos quais os alunos apresentam mais dificuldades em Aritmética e nas operações básicas, sendo que tais dificuldades geralmente se arrastam para o Ensino Médio), interatividade, clareza nas informações visuais, capacidade de motivar e acessibilidade (MEDEIROS e SCHIMIGUEL, 2012; VILARINHO e LEITE, 2015). A base de busca dos aplicativos foi a Play Store, onde foi colocado o string "aplicativos para o ensino de aritmética" e sendo selecionado três aplicativos que apresentassem as características citadas anteriormente: o MathUp, Aventura do Bebê Panda com Matemática e Train Brain.

É importante ressaltar que o uso dos aplicativos para o ensino de conteúdos matemáticos exige planejamento da aula aliando-os aos conteúdos já trabalhados ou aos conteúdos que se pretende desenvolver, ou seja, priorizando a revisão, fixação e assimilação dos conceitos e procedimentos matemáticos, lembrando que os alunos devem compreender que os aplicativos utilizados na sala de aula não podem ser reduzidos à instrumentos de diversão e lazer, mas sim ferramentas auxiliares do processo ensinoaprendizagem e que possuem intencionalidade educativa. 


\section{As potencialidades do aplicativo Mathup para o Ensino de Matemática}

O aplicativo MathUp apresenta um jogo em que o aluno poderá mobilizar suas habilidades cognitivas no que se refere às operações matemáticas, em que a cada nova fase as operações vão ficando mais complexas e desafiadoras. Sabemos que atualmente, a maioria dos cálculos são feitos por dispositivos tecnológicos, como computadores, programas específicos, calculadoras programáveis, dentre outros e, com isso, vários são os caminhos para utilizar essas ferramentas em sala de aula, a depender da forma em que o conteúdo está ministrado.

Para tal, destacamos o que Zabala (1998) apresenta em sua obra: a classificação dos conteúdos factuais, conceituais, procedimentais e atitudinais, para que o professor possa analisar os conteúdos e saber quais serão desenvolvidos em sala de aula, de modo a utilizar-se das práticas pedagógicas apropriadas, inserindo adequadamente os recursos didáticos tecnológicos adequadamente no planejamento de suas aulas.

Nesse sentido, entendemos que o MathUp representa um instrumento potencialmente significativo para o ensino das operações matemáticas, principalmente no Ensino Fundamental, em que muito se utilizam dos conteúdos procedimentais da Aritmética e dos factuais e atitudinais para que os alunos possam resolver problemas aplicados ao cotidiano e utilizando as operações básicas, por exemplo.

O trabalho com as operações presentes nesse jogo corrobora com o que coloca Nascimento (2019, p. 04) acerca da importância das operações matemáticas básicas, visto que irão servir como base para conteúdos futuros, sendo necessário trabalhar a tabuada de modo dinâmico, utilizando recursos que auxiliem na aprendizagem, pois o professor “(...) tem a incumbência de buscar estratégias diversificadas para tornar o processo de aprendizagem mais interessante e prazeroso; dentre as quais cabe destacar a utilização de: materiais concretos, tecnologias digitais, jogos didáticos, dentre outras."

Em complemento ao que foi colocado, a BNCC (BRASIL, 2018, p. 276) faz a uma afirmação que destaca a importância de se desenvolver as habilidades matemáticas relacionadas às operações básicas:

No que diz respeito ao cálculo, é necessário acrescentar, à realização dos algoritmos das operações, a habilidade de efetuar cálculos mentalmente, fazer estimativas, usar calculadora e, ainda, para decidir quando é apropriado usar um ou outro procedimento de cálculo. 
Deste modo, o jogo MathUp será útil para auxiliar o professor a atingir os objetivos propostos pela BNCC (BRASIL, 2018) em relação ao ensino de Matemática no Ensino Fundamental e o desenvolvimento do pensamento aritmético que tem como fundamento principal o sentido de número e as operações matemáticas básicas.

O aplicativo é MatchUP consiste num jogo que pode ser baixado pelo smartphone acessando da loja de aplicativos, disponível para Android e iOS. O idioma do aplicativo é inglês, mas não interfere na acessibilidade porque os comandos são simples e também não interfere na compreensão das operações. Na tela inicial, o aluno poderá iniciar o jogo optando pelo modo fácil ou difícil, além de conseguir ter acesso as configurações do jogo, bem como a opção de observar a sua pontuação.

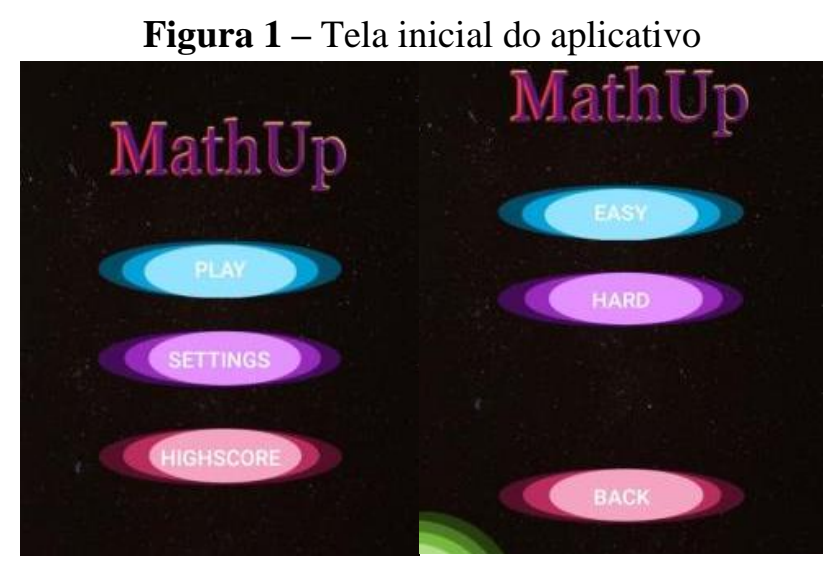

Fonte: Acervo dos Autores (2021).

As regras do jogo também podem ser observadas ao clicar em configurações, onde será informado que o aluno deverá optar entre o modo fácil ou difícil e terá 30 segundos para resolver os exemplos que surgirão na tela; além disso, para que o aluno possa prosseguir para o próximo nível, deverá obter dez ou mais respostas corretas.

A cada nível seguinte, a dificuldade dos exemplos aumentará, e por fim, o aluno poderá apresentar no máximo três respostas incorretas por nível para não ser eliminado do jogo. Na parte superior da tela em cor verde (lado direito) aparecem os acertos e em cor vermelha (lado esquerdo) aparecem os erros. 


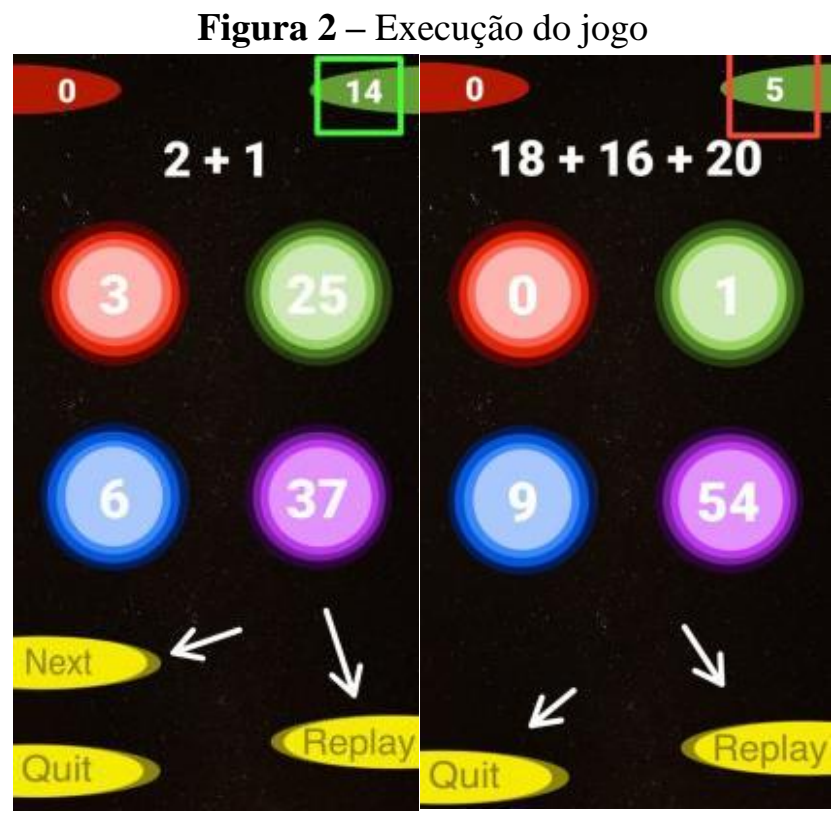

Fonte: Acervo dos Autores (2021).

Nota-se que o jogo proporciona o estudo das operações básicas de modo interativo, lidando com desafios como o tempo, quantidade de acertos e erros, bem como o resultado de seu progresso, fazendo com que o aluno tenha mais interesse em resolver as operações, com o intuito de prosseguir no jogo através de seus acertos e erros, analisando estratégias para saber o motivo de seus erros e superá-los.

As dificuldades apresentadas no jogo a cada nível também é um ponto positivo a se destacar, pois sabemos que os alunos gostam de lidar com materiais dinâmicos, possibilitando uma nova visão de encarar as operações a cada nova fase. Um eventual empecilho para o uso do MathUp no contexto escolar pode se dar por conta do idioma em inglês, porém o professor rapidamente pode explicar aos alunos quais são as regras do jogo, qual o intuito e como prosseguir nas fases que vão surgindo. Deste modo, acreditamos que o jogo MathUp apresenta potencialidades que contribuem com a revisão/fixação das operações básicas, de modo lúdico e de fácil acesso.

\section{As potencialidades do aplicativo "Aventura do Bebê Panda com Matemática"}

Podendo ser baixado na Play Store e estando disponível para o sistema Android e iOS, o jogo Aventura do Bebê Panda com Matemática apresenta um layout bastante atrativo que chama a atenção do aluno, pois o cenário é bastante colorido e possui 
personagens que acompanham o Bebê Panda durante as atividades. Nesse aplicativo, o aluno poderá lidar com diversos tipos de atividades matemáticas, a fim de restaurar uma ilha com dinossauros. O aplicativo pode ser utilizado como recurso pedagógico principalmente na Educação Infantil e anos iniciais do Ensino Fundamental, pois os principais conteúdos matemáticos utilizados se referem à cognição numérica, ao espaço geométrico e a algumas operações básicas, como adição.

Figura 3 - Tela inicial do Aplicativo

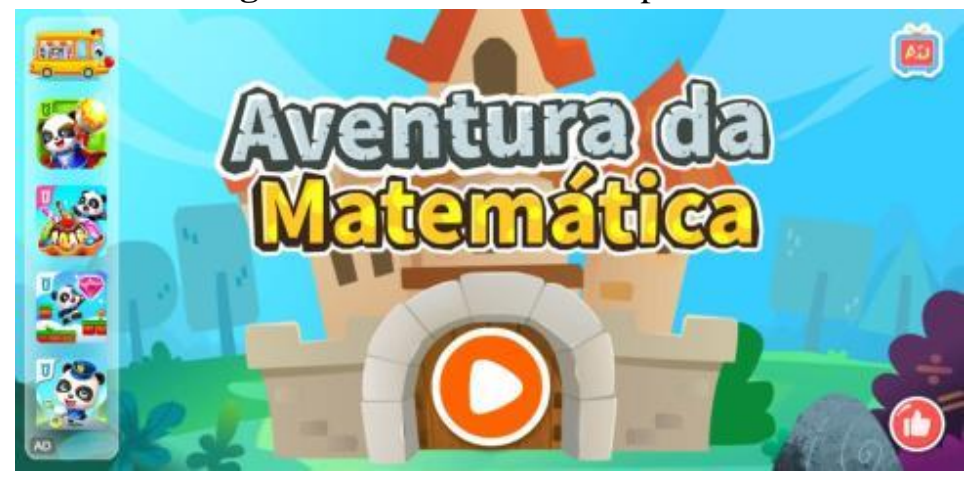

Fonte: Acervo dos Autores (2021).

O professor poderá utilizar o jogo para auxiliar em suas aulas, adaptando atividades que seriam feitas sem o auxílio da tecnologia. O jogo apresenta recompensas ao longo das etapas e o aluno vai progredindo nos conteúdos destacados anteriormente. Observamos que a diversidade e a quantidade de atividades são consideravelmente abrangentes no que diz respeito aos conteúdos abordados:

Figura 4 - Lista de atividades apresentadas no Aplicativos
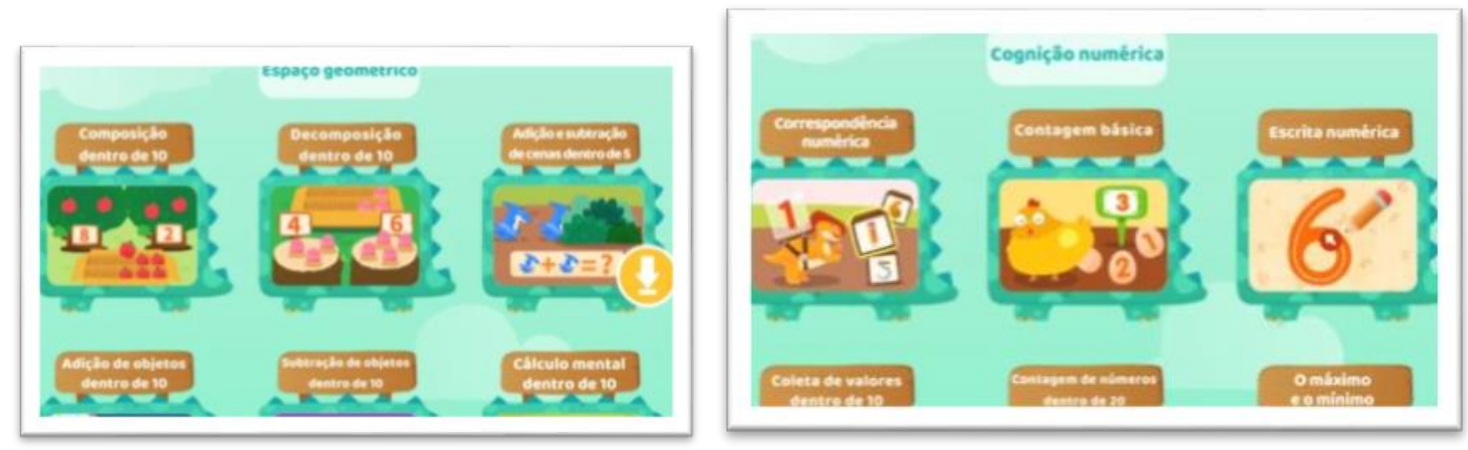

Fonte: Acervo dos Auto0res (2021). 
No que se refere às potencialidades desse jogo, destacamos o fato de que a BNCC (BRASIL, 2018) coloca que na unidade temática "Números" deve-se desenvolver um pensamento numérico "que implica o conhecimento de maneiras de quantificar atributos de objetos e de julgar e interpretar argumentos baseados em quantidades" (BRASIL, 2018, p. 268). Assim, fica evidente que a contagem na qual o aluno associa quantidades ao numeral, é essencial para o desenvolvimento do sentido de número e esse aplicativo é uma ferramenta que auxilia nesse processo.

Deste modo, os outros conteúdos trabalhados, como as operações básicas, também tornam o uso desse jogo relevante para o contexto educacional, visto que os alunos podem desenvolver uma maior compreensão da Matemática através de um jogo que envolve os conteúdos de modo atrativo e com objetivo, visto que a cada conquista, a ilha dos dinossauros vai se expandindo mais e mais. Trata-se de um jogo educativo que envolve conteúdos procedimentais.

Figura 5 - Ilha dos dinossauros e atividade
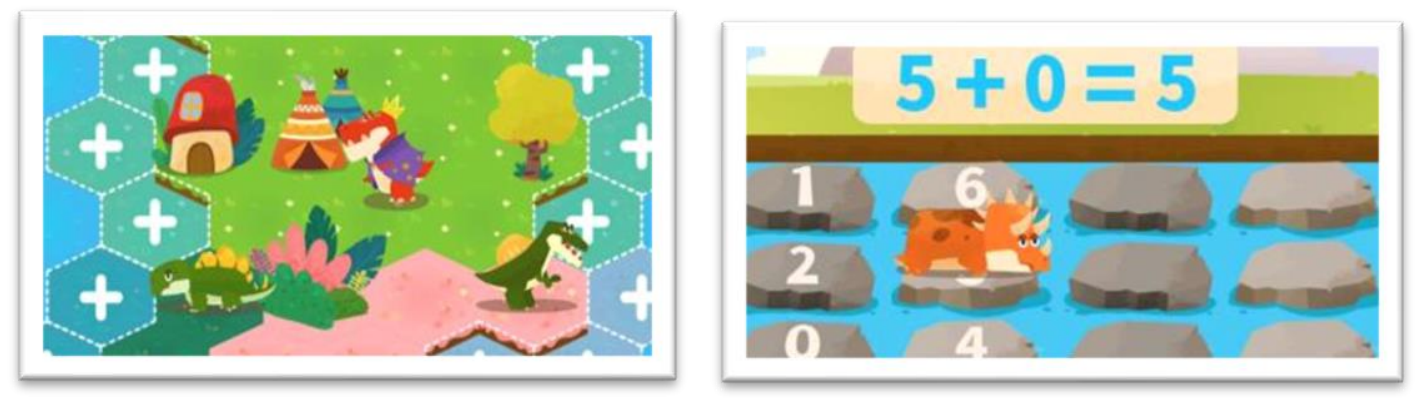

Fonte: Acervo dos Autores (2021)

A empresa BabyBus que é a criadora do aplicativo apresenta outros aplicativos com o personagem Bebê Panda, sendo uns voltados para o ensino de conteúdos escolares como este que analisamos e outros voltados para diversão, constituindo-se exclusivamente como jogos sem intencionalidade educativa.

É imprescindível que o professor teste o jogo previamente, verifique quais atividades estão relacionadas aos conteúdos, habilidades e competências que pretende desenvolver, e assim planeje sua aula, agregando-a com outras atividades que devem ser desenvolvidas após o uso do aplicativo, com caráter diagnóstico para verificar se os conteúdos foram assimilados, por exemplo. 


\section{As potencialidades do aplicativo Train Brain no Ensino de Matemática}

O Train Brain pode ser baixado no smartphone e está disponível para o sistema Android e iOS. É um aplicativo simples que tem como foco a operação multiplicação. Nele, o aluno pode escolher o nível de dificuldade com as parcelas que estão sendo multiplicadas, ambas variando de 2 até o numeral 30 (ver figura 6). Para jogar, o aluno tem inicialmente 3 vidas que lhe permitem errar 3 vezes as operações dadas, operações estas que possuem um tempo para serem respondidas e que o jogador deve escolher a opção certa entre as três alternativas apresentadas pelo aplicativo, como mostra a figura 6.

Ademais, a pontuação do aluno está apresentada pela estrelinha, que ao longo do jogo marca qual a melhor pontuação até aquele momento e o ícone de certo mostra quantas operações o aluno já realizou.

Figura 6 - Início do jogo Train Brain e a operação de multiplicação no jogo

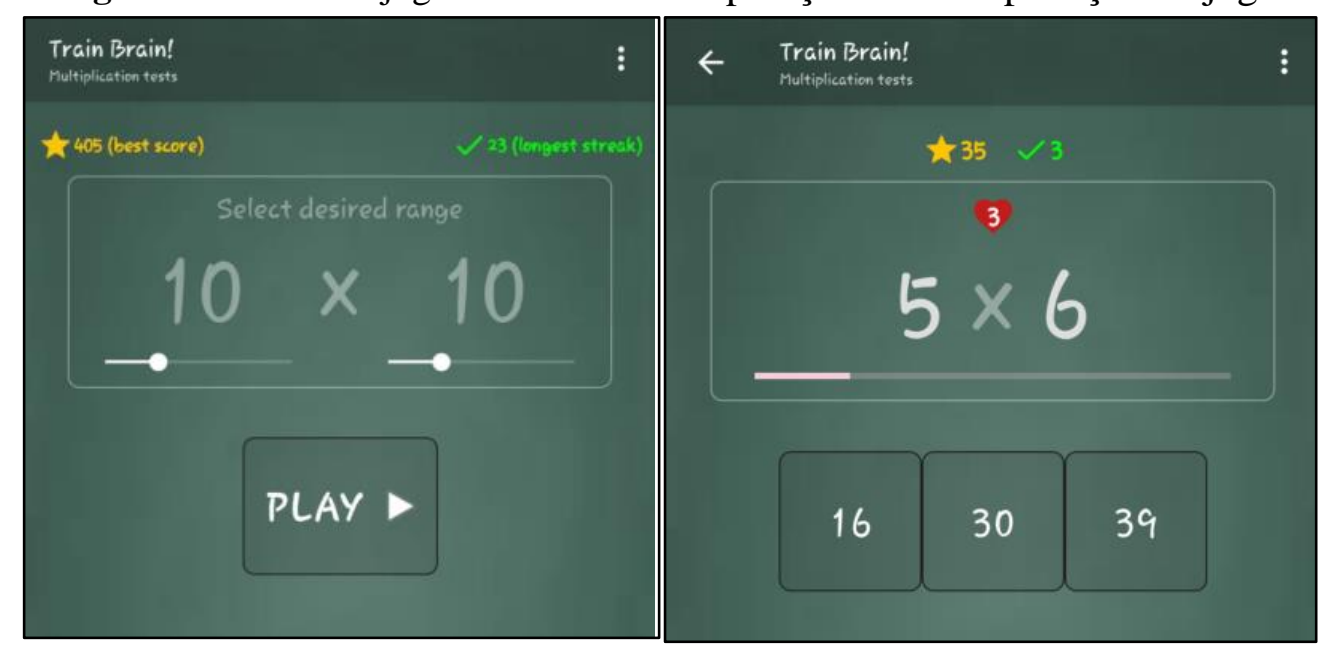

Fonte: Acervo dos Autores (2021)

Ao analisarmos as potencialidades desse aplicativo, retomamos a discussão acerca da necessidade de dinamizar os conteúdos em sala de aula, em particular, os conteúdos que assumem a classificação como conteúdos procedimentais de Zabala (1998). Deste modo, o Train Brain torna o ensino da operação de multiplicação no Ensino Fundamental mais dinâmico, de modo a possibilitar que os alunos desenvolvam habilidades como a agilidade, o raciocínio lógico, além da construção da tabuada de multiplicação. 
A BNCC (BRASIL, 2018) coloca que os jogos digitais e a gamificação em sala de aula são capazes de ressignificar todo o processo de aprendizagem, proporcionando para o aluno um aprendizado mais dinâmico e ativo, além de ser recursivo, possibilitando ao aluno a retomada dos conteúdos a qualquer momento com praticidade de acesso, além de ajudar o aluno a lembrar das estratégias utilizadas para superar os desafios apresentados pelos jogos em sala de aula.

Sobre a gamificação cabe pontuar brevemente que "corresponde ao uso de mecanismos de jogos orientados ao objetivo de resolver problemas práticos ou de despertar engajamento entre um público específico" (VIANNA et al., 2013, p. 15). Em geral, no contexto educacional como explica Kapp (2015) são utilizados dois tipos de gamificação, a estrutural (com utilização de elementos de jogos para promover a aprendizagem como pontuação e placar) e a de conteúdo (criação de um ambiente de jogo na sala de aula para promover a aprendizagem, utilizando histórias, desafios e personagens, levando os alunos a executarem suas ações como em um jogo, pois passam a pensar como se estivessem dentro de um jogo - game thinking). No caso, deste artigo não tratamos especificamente da gamificação que possui outras características além destas citadas, portanto, nossa análise não abrange gamificação, e sim análise das potencialidades dos aplicativos.

Por fim, sobre o Train Brain é importante mencionar que permite ao professor acompanhar de forma integral o processo de assimilação, revisão e fixação da operação multiplicação, desde os anos iniciais quando o aluno constrói a noção de dobro e triplo, até os anos finais do Ensino Fundamental, quando o conhecimento do aluno estará mais sólido para que ele possa realizar as operações com números maiores. Destacamos ainda que o Train Brain instiga e motiva o aluno a treinar mais e mais a multiplicação, fixando as destrezas procedimentais, chegando a níveis mais complexos da própria operação, em que ele baterá os próprios recordes no jogo, realizando cada vez mais novas multiplicações.

\section{Considerações Finais}

Desta forma, a partir do que foi apresentado neste trabalho, constatamos que os três aplicativos, que consistem em jogos, são potencialmente significativos para o ensino 
de Matemática na Educação Infantil e nos anos iniciais do Ensino Fundamental e se caracterizam por enfatizar conteúdos procedimentais, na medida que focam na compreensão dos algoritmos das operações matemáticas básicas. São de fácil acesso e os alunos não apresentarão dificuldades de acesso e manuseio com os jogos, além de se adequarem aos conteúdos que estão sendo ministrados, o que implica em facilidade de sua inserção no planejamento da aula. Além do mais, contribuem para o desenvolvimento do cálculo mental, do raciocínio lógico e de estratégias e procedimentos para resolução das operações matemáticas básicas, que muitas vezes não são despertados com uma aula puramente tradicional, com caráter transmissivo e atividades centradas na utilização de lápis e papel.

Nesse sentido, avaliamos também que o MathUp, A Aventura do Bebê Panda com Matemática e o Train Brain ${ }^{4}$ são ferramentas capazes de inserir o aluno na cultura digital conforme orienta a BNCC (BRASIL, 2018).

Concluímos, portanto, que embora aprender Matemática não seja um processo fácil para muitos, pode ser um caminho menos tortuoso e mais acolhedor a partir do momento em que professor inova suas práticas pedagógicas inserindo recursos que auxiliam no processo de ensino-aprendizagem como as novas tecnologias educacionais, tornando o ensino motivador, dinâmico e atraente para o aluno, reduzindo dificuldades na compreensão dos conceitos matemáticos e também superando-as, e os aplicativos aqui apresentados são uma alternativa nesse sentido.

\section{Referências}

ALBINO, T. S. L. A prática docente e o uso de metodologias alternativas no ensino de matemática: um olhar para as escolas que adotam propostas pedagógicas diferenciadas. Disponível em: https://www.ufjf.br/ebrapem2015/files/2015/10/gd7_thais_albino.pdf. Acesso em: 08 abr. 2021.

\footnotetext{
${ }^{4} \mathrm{O}$ download dos aplicativos pode ser feito através:

Panda Matemática:

https://play.google.com/store/apps/details?id=com.sinyee.babybus.math

MathUp:

https://play.google.com/store/apps/details?id=com.xapp.mathup

Train Brain:

https://play.google.com/store/apps/details?id=com.mastercluster.trainbrain\&hl=pt
} 
ALMEIDA, H. M. O uso de celulares, tablets e notebooks no ensino da matemática. Revemat. Florianópolis (SC), v.11, n. 2, p. 318-327, 2016.

BOGDAN, R. C.; BIKLEN, S. K. Investigação qualitativa em educação. Porto: Porto Editora, 1994.

BRASIL. Parâmetros curriculares nacionais. Brasília, DF: MEC/SEF, 1998. Básica, 2018.

Base nacional comum curricular. Brasília: MEC/Secretaria de Educação

D'AMBROSIO, U. História da Matemática e Educação. Cadernos CEDES no 40. Campinas, SP: Papirus,1996. p. 7-17.

EYYAM, R.; YARATAN, H. S. Impact of use of technology in mathematics lessons on student achievement and attitudes. Social Behavior and Personality. v. 42, n.1, p. 3142, 2014.

KAPP, K. An elusive definition: gamification for learning. Disponível em: <https://www.linkedin.com/pulse/elusive-definition-gamification-learning-karlkapp>. Acesso em: 10 abr. 2021.

KYRIAKIDES, A.O., MELETIOU-MAVROTHERIS, M.; PRODROMOU, T. Mobile technologies in the service of students' learning of mathematics: the example of game application A.L.E.X. in the context of a primary school in Cyprus. Mathematics Education Research Journal, v. 28, p. 53-78, 2016.

LORENZONI, M. Pequeno glossário de inovação educacional. São Paulo: Geekie, 2016.

MEDEIROS, M., SCHIMIGUEL, J. Uma abordagem para avaliação de jogos educativos: ênfase no ensino fundamental. Revista Novas Tecnologias na Educação, (Renote), v.10, n.3, 2012.

MORAN, J. M. Ensino e aprendizagem inovadores com tecnologias. In: MORAN, J.M.; MASETTO, M. T.; BEHRENS, M.A.(Orgs). Novas tecnologias e mediação pedagógica. $21^{\mathrm{a}}$ ed. Campinas: Papirus, 2013. p. 11-66.

MORETTO, T. C. As tecnologias móveis no ensino de Matemática. 2015. $101 \mathrm{f}$. Dissertação (Mestrado em Educação) - Universidade de Lisboa, Lisboa, 2015

NASCIMENTO, F. M. O uso do Math Duel no processo de aprendizado das operações básicas de matemática no $6^{\circ}$ ano do ensino fundamental. Disponível em: < http://repositorioinstitucional.uea.edu.br/handle/riuea/3100 $>$. Acesso em: $18 \mathrm{fev}$. 2021. 
Ewellyn Amâncio Araújo Barbosa, Sidney Leandro da Silva Viana e Claudia de Oliveira Lozada Uma análise das potencialidades dos aplicativos Mathup, aventura do bebê panda com matemática e Train Brain para o ensino das operações básicas na educação infantil e anos iniciais do ensino fundamental

OSMUNDO, L. A BNCC e a cultura digital. São Paulo: Instituto Singularidades, 2018.

REIS, J. B. A. O conceito de tecnologia e tecnologia educacional para alunos do ensino médio e superior. Disponível em: http://alb.org.br/arquivo-

morto/edicoes_anteriores/anais17/txtcompletos/sem16/COLE_932.pdf. Acesso em: 18 fev. 2021.

VIANNA, Y. et al.. Gamification, Inc.: Como reinventar empresas a partir de jogos, Rio de Janeiro: MJV Press, 2013.

VILARINHO, L. R. G.; LEITE, M. P. Avaliação de jogos eletrônicos para uso na prática pedagógica: ultrapassando a escolha baseada no bom senso. Revista Novas Tecnologias na Educação, (Renote), v.13, n.1, 2015.

ZABALA, A. A prática educativa: como ensinar. Porto Alegre: ArtMed, 1998. kind of app using in math education.

Recebido em: 08 / 03 / 2021

Aprovado em: 23 / 04 / 2021 